\title{
Algunos apuntes acerca de la contribución del Profesor Manuel Calvo a la introducción y consolidación de la sociología jurídica en España

\author{
(Some notes on Professor Manuel Calvo's contribution to the introduction \\ and consolidation of Sociology of Law in Spain)
}

OÑATI SOCIO-LEGAL SERIES VOLUME 11, ISSUE 3 (2021), 605-622: INVESTIGATIONS - INVESTIGACIONES

- IKERLANAK

DOI LINK: HTTPS://DOI.ORG/10.35295/OSLS.IISL/0000-0000-0000-1190

RECEIVED 26 APRIL 2021, ACCEPTED 19 MAY 2021

JORGE GRACIA IBÁÑEZ*

\section{Resumen}

En este artículo, en homenaje a la figura del Catedrático de Filosofía del Derecho y Sociología Jurídica Manuel Calvo García de la Universidad de Zaragoza, se realizan algunos apuntes y consideraciones acerca de su contribución a la introducción y consolidación de la sociología jurídica en España. En primer lugar, se señalan algunos aspectos destacados acerca de sus aportaciones teóricas sobre la naturaleza y el papel de la sociología jurídica en el estudio y análisis del derecho como fenómeno social. Después, se describen algunas de sus contribuciones en el campo desde dos espacios institucionales y académicos: el Instituto Internacional de Sociología Jurídica de Oñati y el Laboratorio de Sociología Jurídica de la Universidad de Zaragoza. Finalmente, se analizan algunos aspectos de su trabajo, tanto desde el punto de vista teórico como empírico, en relación con la violencia de género, que ejemplifican su concepción de una sociología jurídica crítica y transformadora.

\section{Palabras clave}

Manuel Calvo; sociología jurídica; investigación socio-jurídica; violencia de género

Quiero agradecer las sugerencias e informaciones proporcionadas para la elaboración de este artículo a las Profesoras Teresa Picontó, María José González Ordovás, María José Bernuz, al Profesor Daniel Oliver Lalana y a Carmen Mesa Raya.

* Laboratorio de Sociología Jurídica de la Universidad de Zaragoza/Interdisciplinary Research Center on Crime, Justice and Security (CJS), School of Criminology, University of Porto (Portugal). Dirección de email: jibanez@direito.up.pt 


\begin{abstract}
In this article in homage to the figure of the Professor of Philosophy of Law and Sociology of Law Manuel Calvo Garcia from University of Zaragoza, some notes and considerations are made about his contribution to the introduction and consolidation of Sociology of Law in Spain. First, some aspects are pointed out about his theoretical contributions on the nature and role of Sociology of Law in Oñati in the study and analysis of law as a social phenomenon. Then, some of his contributions in the field are described from two institutional and academic spaces: The International Institute for the Sociology of Law and the Laboratory of Sociology of Law in the University of Zaragoza. Finally, some aspects of his body of work are analyzed, both from a theoretical and an empirical point of view, in relation to gender violence that exemplify his conception of a critical and transformative Sociology of Law.
\end{abstract}

\title{
Palabras clave
}

Manuel Calvo; Sociology of Law; Socio-legal research; gender violence 


\section{Table of contents}

1. Introducción: pensando a Manuel Calvo García 608

2. Tres opciones epistemológicas: teoría sociológica del derecho, jurisprudencia sociológica y sociología jurídica empírica 608 3. Dos hitos, dos espacios: el Instituto de Sociología Jurídica de Oñati y el Laboratorio de Sociología Jurídica de la Universidad de Zaragoza

4. La violencia de género como prioridad en la investigación socio-jurídica..................617

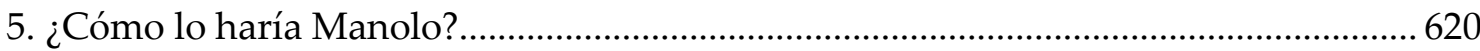

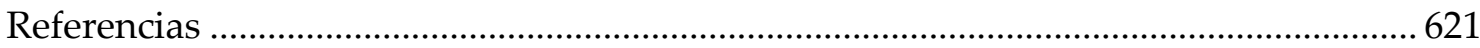




\section{Introducción: pensando a Manuel Calvo García}

Resulta difícil abordar un artículo como este por diferentes razones. La fundamental es que, inevitablemente, solo puede hacerse desde la constatación de la pérdida que significa la desaparición de una persona que fue una referencia tanto en lo personal como en lo académico. Pero también porque las facetas del trabajo y la contribución intelectual de Manuel Calvo abarcan muchos aspectos y resulta necesario escoger entre ellos.

Por eso he elegido reordenar y dar alguna estructura a estos apuntes para convertirlos en una forma de recordar al Profesor Manuel Calvo con la que creo estaría básicamente de acuerdo. En primer lugar, centrándome en algunos aspectos de su aportación intelectual en el ámbito de la sociología jurídica. En segundo lugar, más específicamente, dirigiendo mi análisis hacia su contribución en la introducción y consolidación de la disciplina en España. Para ello, me detendré en rememorar algunos momentos clave en su relación con la sociología jurídica, que tuvieron como escenario dos espacios académicos muy queridos por él: el Instituto Internacional de Sociología Jurídica de Oñati (IISJ), del que fue director científico durante unos años, y el Laboratorio de Sociología Jurídica de la Universidad de Zaragoza (LSJUZ), que fue creado, impulsado y dirigido por él hasta el momento de su fallecimiento. En tercer y último lugar, dedicaré un espacio a reflexionar acerca de algunas de las aportaciones teóricas y empíricas en relación con la violencia de género, una de sus principales líneas de investigación ya que, los numerosos e importantes trabajos que dirigió y lideró en ese ámbito, ejemplifican su concepción de la sociología jurídica.

Algunos compañeros y amigos colombianos utilizan para decir que se acuerdan de nosotros, que nos echan de menos cuando estamos ausentes, una curiosa expresión:le pienso. Creo que ese modismo explica bien la finalidad de estas páginas, que no es otra que la de pensar al Profesor Manuel Calvo. Esto es, otra forma de expresar cuánto lo echamos de menos.

\section{Tres opciones epistemológicas: teoría sociológica del derecho, jurisprudencia sociológica y sociología jurídica empírica}

En su concepción de la sociología jurídica, Manuel Calvo siempre tuvo presente la idea de que esta nunca ha representado una perspectiva unitaria, reconociendo al menos tres opciones o planteamientos típicos: la teoría sociológica del derecho, la jurisprudencia sociológica y la sociología empírica del derecho (Calvo 1995, 18).

La primera opción epistemológica, la teoría sociológica del derecho, entroncaría remotamente con el estudio que realizaron del derecho los autores clásicos de la ciencia social. Algunos de esos autores (Gumplowicz, Weber y Durkheim, entre otros) atribuyeron a las funciones del derecho una gran importancia en la estructuración del orden social, ya fuese "como instrumento de dominación o como instrumento de cohesión social" (Calvo 1995, 19). Pero, pese al interés inicial, el Profesor Calvo consideraba que los sociólogos posteriores lo ignoraron casi todo sobre el derecho con excepción de la atención prestada por el marxismo y algunas sociologías conflictualistas. La crisis teórica interna y externa- del funcionalismo habría revalorizado el peso del derecho en la teoría social (Calvo 1995, 19). 
La teoría tradicional del derecho se asentaba en postulados formalistas que desatendían todo lo que no fuera un concepto técnico del mismo y el estudio de su estructura. Para el Profesor Calvo (1995, 19-20), ya desde mediados de los 90, las preguntas sobre qué es el derecho habían perdido preponderancia y eran sustituidas por preguntas sobre cómo funciona el derecho. Como consecuencia de ese desplazamiento y de la revalorización del derecho en la teoría social, surge un espacio que, precisamente, vincula teoría social y derecho; es decir, un espacio para la teoría sociológica del derecho.

El antiformalismo jurídico a principios del siglo XX había dado entrada a la sociología en la ciencia jurídica, propiciando a la larga el desarrollo de un segundo planteamiento típico: la jurisprudencia sociológica. El derecho vivo o el derecho en acción representa, según su valoración, "una de las características más importantes de las corrientes antiformalistas y al mismo tiempo uno de sus logros más significativos" (Calvo 1995, 20). Entre los referentes teóricos, el Profesor Calvo siempre tuvo muy presentes la obra de Petrazycki, Ehrlich y -en el ámbito del realismo jurídico americano, aunque en sintonía con el antiformalismo europeo- los trabajos del juez Oliver Wendell Holmes que abrieron paso a la jurisprudencia sociológica de Roscoe Pound o las posiciones más estrictamente realistas de Llewellyn y Frank. Veía también una prolongación de esas corrientes -que, simplificando algo las cosas, podríamos denominar "realismo jurídico americano" - en otras, como el círculo de la Law \& Society Science y los Critical Legal Studies (Calvo 1995, 21). De esta forma, "al corregir los excesos del formalismo, la jurisprudencia sociológica puede tener un interés práctico y sintoniza con la tímida orientación de la dogmática hacia las ciencias sociales" (Calvo 1995, 21).

La tercera opción epistemológica que el profesor Calvo (1995, 22 y ss.) identificaba, a mediados de los 90, era la sociología empírica del derecho, que apuesta por recurrir a los métodos y técnicas de la investigación social con el fin de propiciar un conocimiento empírico de los temas jurídicos. La sociología general con posterioridad pareció identificarse con un modelo americano, centrado en el desarrollo de técnicas y procedimientos adecuados para el conocimiento empírico de las realidades sociales, contrapuesto al modelo europeo, más orientado hacia la teoría social. La sociología empírica del derecho se desarrolla, en la mayoría de los países europeos, en pleno auge del positivismo sociológico, de la americanización de la sociología mundial (Treves 1988, 134) y -a pesar de la importancia inicial de los métodos cualitativos- del reinado casi incontestable de los métodos cuantitativos. De hecho, la superación de esta dicotomía excluyente entre lo cuantitativo y cualitativo será, como veremos después, uno de los rasgos de la sociología jurídica tal y como era entendida por Manuel Calvo.

En la formación de ese entendimiento, desde luego, fue fundamental el magisterio de Renato Treves, que propugnaba el desarrollo de una nueva sociología del derecho, como, "aquella constituida por las investigaciones empíricas realizadas sobre los hechos particulares y sobre los problemas sectoriales que interesan grandemente a la vida y al desarrollo de la sociedad contemporánea"' (Treves 1988, 122).

El viaje del Profesor Calvo a Milán en 1988, con el fin de acercarse a la sociología del derecho de la mano del propio Renato Treves y también de Ferrari, fue fundamental, como él mismo reconoció en el elogio pronunciado en el acto de investidura como Doctor Honoris Causa del Profesor Vincenzo Ferrari por la Universidad de Zaragoza en 2014. Así como fue también inspirador el Congreso Internacional de Sociología Jurídica 
celebrado en la Universidad de Bolonia con motivo del 900 aniversario de esa universidad organizado por Ferrari. Manuel Calvo reconoce que tanto la estancia en Milán como ese congreso abrieron las puertas de su orientación hacia la sociología jurídica (Calvo 2014, 8-9). La influencia italiana es insoslayable en su concepción de la sociología jurídica que aúna tanto la dimensión empírica como la teórica. En este sentido -y a pesar de reconocer, en la senda del magisterio de Treves, el peso relevante de la investigación empírica- para Manuel Calvo, la sociología jurídica nunca se quedó solo en lo empírico, admitiendo que la orientación crítica de la misma "requería la profundización teórica en las cuestiones socio-jurídicas" (Calvo 2014, 14).

Él mismo había descrito la investigación socio-jurídica en el ámbito de la sociología del derecho española como una realidad "exigua, dispersa y cuestionada" (Calvo 1995, 17). Manuel Calvo considera que "este fundamento empírico [de la sociología jurídica] no quiere decir que todos los investigadores en el ámbito socio-jurídico deban hacer trabajo de campo, sino que tiene que haber personas y grupos que afronten esta tarea y cauces para fomentar el diálogo entre quienes la hacen y quienes trabajan en el terreno del análisis o la reflexión teórica" (Calvo 2010, 391).

En este sentido, se apartaba de la tesis de Renato Treves (1988), para quien sólo cabía el fundamento empírico para la Sociología Jurídica. El Profesor Calvo (2010, 392), más flexible en este punto, alega que la experiencia le ha hecho ver "que no todos los enfoques de la sociología jurídica pueden reducirse a la sociología empírica del derecho que Treves propugnaba y que no todos los investigadores en este campo tienen interés o condiciones para hacer investigación empírica". En cualquier caso, como veremos después, fue siempre coherente con esa concepción de la sociología jurídica, fomentando y dirigiendo a través del Laboratorio de Sociología Jurídica de Zaragoza, numerosos trabajos empíricos sobre diferentes temas. ${ }^{1}$

Resulta esclarecedor, en el análisis de sus aportaciones teóricas a la materia, el artículo en el que Manuel Calvo se preguntaba: ¿Cabe el enfoque socio-jurídico en la teoría del Derecho? (Calvo 2010). En ese trabajo, el Profesor Calvo defiende la posibilidad de la existencia de diversos enfoques para reflexionar teóricamente sobre el Derecho y que, al menos algunos de ellos que no resulten antagónicos se pueden complementar. Pero como advierte, "no puede pretenderse que todo vale". De esta forma:

Caben diversas teorías del derecho, pero debe exigirse que sean teorías válidas en orden a alcanzar los objetivos de una construcción teórica: deben ser útiles desde un punto de vista práctico y facilitar la compresión de los fenómenos jurídicos entendidos en un sentido amplio y plural. Una teoría filosófica basada en valores de verdad sería en principio una teoría válida; pero no a costa de limitar su perspectiva y utilidad como ocurre con los planteamientos más estrechos de la teoría analítica y, en cualquier caso, se deberá admitir que este enfoque es discutible. De la misma manera, una teoría crítica no tiene por qué ser rechazada a priori o tachada sin más de ideológica (...). Algo diferente es que, para ser considerada una teoría válida, deberá superar algunos test que permitan comprobar, por una parte, si facilita la explicación y comprensión de los fenómenos jurídicos en uso entendidos en sentido amplio y plural y, por otra, su utilidad

\footnotetext{
${ }^{1}$ Entre estos temas podemos destacar el acceso a la justicia de los inmigrantes, políticas de conciliaciónde la vida familiar y profesional, maltrato hacia las personas mayores, seguridad pública, justicia demenores, derechos sociales o participación ciudadana.
} 
desde un punto de vista práctico y enraizado en el funcionamiento efectivo de las instituciones jurídicas (Calvo 2010, 390).

En esta línea reconoce que cabe el enfoque socio-jurídico de la teoría del derecho e incluso, yendo más allá, una teoría socio-jurídica del derecho que "profundice en la descripción y comprensión del derecho como fenómeno social aunando herramientas metodológicas de los dos campos" (Calvo 2010, 392). De esta forma, concluye que:

... la Teoría socio-jurídica del derecho podría ser vista como un punto de encuentro para facilitar el diálogo entre perspectivas y áreas científicas diversas. Esto es, como una aproximación al conocimiento del fenómeno y los procesos del Derecho que bebe en fuentes plurales y propicia el intercambio de resultados entre opciones epistemológicas que se incardinan en diferentes campos del conocimiento -jurídico o no jurídico-, permitiendo así la conjunción de las ideas jurídicas o aproximaciones doctrinales al derecho con los métodos y las perspectivas de las ciencias sociales. (Calvo 2010, 392)

No obstante, el Profesor Calvo era consciente de que, si bien los enfoques teóricos en el ámbito socio-jurídico gozaban de relativa buena salud, las investigaciones empíricas "adolecen de una clara debilidad y serían de todo punto insuficientes, tanto desde el punto de vista de las funciones auxiliares al desarrollo y funcionamiento del derecho como desde las posibles aportaciones al desarrollo de una teoría del derecho enfocada sociojurídicamente" (Calvo 2010, 392).

Algunas de los rasgos asociados a su concepción de sociología jurídica que ya aparecen en obras anteriores, fueron sistematizados y concretados en un texto posterior, junto con Teresa Picontó (Calvo y Picontó 2017). La sociología jurídica así entendida puede caracterizarse por cuatro rasgos esenciales. Siguiendo a Calvo y Picontó (2017), esos rasgos podrían sintetizarse de la siguiente manera:

La sociología jurídica es un punto de encuentro interdisciplinario. Si los contenidos de la sociología jurídica son plurales (incluyendo aquí cuestiones relacionadas con los derechos humanos), es posible decir que los perfiles de la sociología jurídica son porosos. Hasta el punto de que resulta difícil establecer barreras "disciplinares" entre la sociología jurídica y otros campos de investigación vinculados a la criminología, la ciencia política, las ciencias de la administración, la antropología jurídica, la psicología jurídica, etc. Por eso mismo,la sociología jurídica podría ser vista como una disciplina que facilita el diálogo entre áreas científicas diversas y que se acerca al conocimiento del fenómeno y las dinámicas del derecho bebiendo en fuentes plurales y propiciando el intercambio de resultados entre disciplinas que se incardinan en diferentes campos del conocimiento -jurídico o no jurídico-, permitiendo así, por una parte, la conjunción de los métodos y las perspectivas de las ciencias sociales y jurídicas y, por otra, el intercambio con las disciplinas fronterizas.

La sociología jurídica está sustentada en investigaciones empíricas. A pesar de que la sociología jurídica admite enfoques plurales, algunos de ellos centrados en dimensiones teóricas y críticas, en un sentido general, la investigación socio-jurídica debe sustentarse en investigaciones empíricas, tanto cuantitativas como cualitativas. Este uso conjunto de las diferentes técnicas de las ciencias sociales debe ayudar a evitar una cierta renuncia metodológica en la que suelen incurrir los juristas por carencias respecto al dominio de las técnicas y métodos de investigación de las ciencias sociales, como la renuncia a la complejidad del objeto de investigación en la que suelen incurrir los sociólogos por la 
falta de conocimiento de las prácticas jurídicas. La pluralidad metodológica debería incentivar además la apertura temática de la disciplina abarcando también muchas cuestiones conectadas con los derechos humanos.

La sociología jurídica exige la vinculación entre investigación empírica y perspectivas teóricas y críticas. Aunque el punto de partida sea empírico, la investigación empírica no debe oscurecer la reflexión y el análisis teórico. Esta vinculación lo teórico y lo empírico es una exigencia epistemológica, pero también forma parte de una apuesta global por una sociología jurídica que no se desarrolle de espaldas a la crítica de las formas jurídicas.

La sociología jurídica tiene vocación reflexiva. Una sociología jurídica encerrada en sí misma carecería de fundamento y de razón de ser. Por eso mismo, es necesario que la sociología jurídica sea reflexiva, es decir, es necesario que ella mantenga una comunicación efectiva con las instituciones y los agentes colectivos e individuales que intervienen en el ámbito de la promoción e implementación de las formas legales. Al fin y al cabo, el saber sociojurídico procede de los agentes sociales y jurídico-institucionales y, por lo tanto, debe hacer revertir los resultados de investigación sobre la praxis. Esto resulta especialmente relevante cuando estamos hablando de objetos de estudio y análisis conectados con los derechos humanos y su potencial emancipador.

En definitiva, como el propio Manuel Calvo, resumía en el mencionado elogio que pronunció en el acto de investidura como Doctor Honoris Causa del Profesor Vincenzo Ferrari por la Universidad de Zaragoza en 2014, la perspectiva a la que se adhería conjuga "una sociología jurídica construida desde el derecho, orientada a la investigación empírica y con el complemento de la teoría socio-jurídica como presupuesto necesario para construir una sociología jurídica crítica" (Calvo 2014, 14).

\section{Dos hitos, dos espacios: el Instituto de Sociología Jurídica de Oñati y el Laboratorio de Sociología Jurídica de la Universidad de Zaragoza}

Resulta revelador partir del balance que Manuel Calvo hacía a mediados de los años 90 de la situación de la sociología jurídica en España, a la hora de percibir, no solo la evolución posterior, sino el alcance de su propia aportación a la consolidación institucional y posterior desarrollo científico de la disciplina en ese mismo contexto estatal. ${ }^{2}$ Para el Profesor Calvo $(1995,26)$, el panorama de la sociología jurídica en España reproducía entonces, aunque bastante imperfectamente, las tres opciones epistemológicas anteriormente comentadas, existiendo algunos destacables trabajos de teoría sociológica del derecho, algunos otros que, aunque como él mismo apostillaba "con algo de imaginación", podrían denominarse jurisprudencia sociológica y, por último, una muestra entonces todavía exigua de investigaciones empíricas. ${ }^{3}$ También apuntaba en su análisis hacia las causas de esa falta de despegue de la investigación sociojurídica en España que no parecían frenar en la misma medida ni a la teoría ni a la

\footnotetext{
2 Aunque nos centremos en este trabajo en la figura de Manuel Calvo resulta de justicia reconocer en este punto la labor de un conjunto de académicos y académicas como Manuel Atienza, Ramón Soriano, Caballero-Harriet, Juan Pérez Lledó, Javier de Lucas, María José Fariñas Dulce, María José Añón, Encarna Bodelón y otros/as que contribuyeron decisivamente con su trabajo a la consolidación de la sociología jurídica en España en sus fases iniciales y con posterioridad.

${ }^{3}$ Por ejemplo, algunos de los trabajos de investigación sobre el contexto social de los jueces y las actitudes de los españoles ante la administración de justicia de Toharia $(1975,1988,1994)$.
} 
jurisprudencia sociológica, pero que impedían un desarrollo coherente de la misma (Calvo 1995, 29). En primer lugar, identificaba que la dialéctica academia-mercado habría tenido un efecto especialmente negativo al disuadir a los jóvenes investigadores de empezar su carrera con trabajos de sociología del derecho. En segundo lugar, consideraba como la investigación institucional no podía tirar por si sola de la investigación sociojurídica por centrarse esencialmente en necesidades políticas de información y focalizarse casi exclusivamente en encuestas de opinión. En tercer lugar, le preocupaba tanto la pobreza metodológica de la sociología del derecho de los juristas como la falta de objetivos de los sociólogos para abrir la investigación socio-jurídica más allá de los temas tópicos. Finalmente, en cuarto y último lugar, recordaba, con cierta ironía, cómo resulta mucho más cómodo trabajar en despachos con aire acondicionado y calefacción que adentrarse en los laberintos burocráticos de los proyectos de investigación que, además, si tienen éxito, desembocan en los inconvenientes de un trabajo de campo (Calvo 1995, 30).

A pesar de estas dificultades descritas, su perspectiva acerca del desarrollo de la sociología jurídica en España era positiva. Tal vez porque el profesor Calvo era una persona estructuralmente optimista -como tantas veces le oí autodefinirse-, pensaba que sólo se podía ir a mejor. Y así, aun a la espera de la necesaria institucionalización académica de la sociología jurídica, ${ }^{4}$ consideraba que había otro tipo de actuaciones, más a mano, que se podían realizar al requerir sólo del compromiso personal (cursos, seminarios, intercambio de conocimientos entre juristas y sociólogos...). Especialmente daba relevancia a la necesidad de crear equipos multidisciplinares en los que, a partir de la colaboración de sociólogos y juristas, estos aprendieran la lógica de lo social y los métodos y técnicas de investigación apropiados para conocer la dimensión social del derecho y aquellos adquirieran la lógica y el imaginario de lo jurídico para investigar los fenómenos ligados al mundo del derecho en toda su complejidad (Calvo 1995, 39). Como concluía, con cierta retranca, hacían falta "juristas y sociólogos dispuestos a colaborar, profesores de derecho renegados o dispuestos a trabajar por las tardes en el campo de la Sociología Jurídica (...) y, sobre todo, jóvenes dispuestos a realizar trabajos de investigación socio-jurídica como tesis doctorales" (Calvo 1995, 39).

A la luz de esta entusiasta llamada a ponerse manos a la obra, gana en sentido analizar, aunque sea brevemente, algunos aspectos de la labor del Profesor Manuel Calvo en dos espacios académicos diferentes, pero muy queridos por él: el Instituto Internacional de Sociología Jurídica de Oñati (IISJ) y el Laboratorio de Sociología Jurídica de la Universidad de Zaragoza (LSJUZ).

Ya en ese temprano análisis de los 90, consideraba Manuel Calvo que la creación del IISJ era una de las principales razones para fundamentar en la realidad el optimismo en torno al futuro de la disciplina debido al eco de sus actividades y a la posibilidad de mantener contactos y organizar seminarios con especialistas de todos los países (Calvo 1995, 39). Y, con el tiempo, esa importante labor del IISJ se ha ido asentando e intensificando, con programas de reuniones y talleres, visitantes de estudio, publicaciones e intercambio de publicaciones con un buen número de universidades y centros de investigación del mundo. La biblioteca/centro de documentación se ha ${ }^{4}$ En la Universidad de Zaragoza la sociología jurídica aparece en el programa de estudios en el curso 2002-
2003, siendo impartida en ese primer curso por la Profesora Teresa Picontó. 
convertido en un referente mundial de primer orden para los académicos, profesores y alumnos interesados en la sociología jurídica.

Es necesario destacar el gran número de investigadores/as que han sido formados a través del Máster en Sociología Jurídica de Oñati, ${ }^{5}$ ya que pone el acento en algunos de los aspectos que el Profesor Calvo encontraba ineludibles para el desarrollo de la Sociología jurídica: la formación de jóvenes académicos y el intercambio de perspectivas. Como recuerdan Susana Arrese y Rogelio Pérez-Perdomo, ya en la ceremonia de inauguración del IISJ en diciembre de 1988, Renato Treves "expresó su confianza en que el Instituto establecería relaciones con los principales centros de estudio y de investigación de Europa y América, con los países latinoamericanos, con el Japón, la India y los países del tercer mundo y que en los trabajos del Instituto participarían profesores y jóvenes graduados provenientes de los más diferentes países" (Treves 1989, 138, cit. in Arrese y Pérez-Perdomo 2016, 904).

El Profesor Manuel Calvo García, tuvo la ocasión de vivir todo esto en primera persona, ya que fue codirector científico del IISJ junto con el Profesor William Felstiner en los años 2000-2003 y también participó como docente en el Máster de Sociología Jurídica de Oñati. A este último respecto, debemos tener en cuenta que, como bien recuerdan Melville y Arrese $(2016,610)$ :

Teaching on the program is seen to be a mark of recognition of the international standing of a scholar, and positions on the program are highly sought.

Y lo que es aún más relevante, como se desvela en un análisis realizado en 2016 en torno a las percepciones de algunos docentes participantes:

It appears that for many teachers, the IISL does not simply assist in the global dissemination of knowledge, but also plays a key role in ensuring the globalization of sociology of law. Teachers praised the IISL for 'fostering' and 'developing' an international sociology of law community that consists of critical, reflective and selfaware scholars and practitioners. For instance, for one teacher the greatest benefit of teaching at the IISL was that it provided an opportunity '...to create a community of young scholars who question and debate the role of law in society'. (Melville y Arrese 2016, 623)

De esta forma, a partir de este análisis, se percibió también cómo:

... many teachers appeared enriched by their experiences of dealing with cultural diversity. They attempted to engage in genuine dialogue with students, drew on multiple perspectives to challenge hegemonic understandings of sociology of law, and interacted with students beyond the walls of the classroom. In this way, teaching at the IISL is as a form of engaged pedagogy which produces cosmopolitan citizens. (Melville y Arrese 2016, 627)

\footnotetext{
${ }^{5}$ Según la sintética descripción de Melville y Arrese (2016, 610): “The Master's degree is a one-year program, taught in English, and awarded as an official Master of Arts degree of the University of the Basque Country. The program has two major components: twelve intensive coursework units; and an individual research dissertation. The program is highly innovative, in that rather than having any in-house teachers, the program is delivered by leading scholars from around the world. This unique format means that students are exposed to outstanding international scholars, rather than the program being limited to teachers that are available within an institution."
} 
Esta perspectiva indudablemente concuerda con las esperanzas que Manuel Calvo manifestaba ya a mediados de los 90 (Calvo 1995) en el papel que iba a tener el IISJ en el desarrollo de la sociología jurídica, especialmente cubriendo la necesidad de formar jóvenes académicos/as en la especialidad, al tiempo que demuestra el gran camino recorrido para hacer realidad la visión de Treves de Oñati "como una ventana abierta al mundo". Al cumplimiento de esa visión se dedicó Manuel Calvo con entusiasmo desde las posiciones de docente y de codirector científico contribuyendo en la tarea de "establecer lazos con la universidad española y latinoamericana, e impulsando a su vez una colaboración muy estrecha con el sistema de justicia tanto estatal como autonómico" (IISJ 2020).

Por eso, con la intención de predicar con el ejemplo en el fortalecimiento de una sociología jurídica empírica, tuvo un papel esencial la creación del Laboratorio de Sociología jurídica de la Universidad de Zaragoza (LSJUZ). No solo bastaba con la introducción de la sociología jurídica como una optativa en muchos planes de estudio de las facultades de derecho, sino que era necesario ser más ambicioso. El LSJUZ fue creado en su momento para coordinar los trabajos de un grupo de investigadoras e investigadores de la Universidad de Zaragoza y de otras universidades. ${ }^{6}$ Con posterioridad, el equipo de investigación de la Universidad de Zaragoza ha merecido el reconocimiento de Grupo Consolidado de Investigación por parte del Gobierno de Aragón en las convocatorias desde 2002 hasta la actualidad. Hay que destacar igualmente que forma parte de la Red temática El Tiempo de los Derechos, en la que se integran 15 grupos de investigación de diferentes universidades españolas ${ }^{7}$ y que pretende promover acciones estratégicas capaces de marcar un punto de inflexión y propiciar un salto de calidad en la investigación jurídica en derechos humanos.

El liderazgo y el impulso del Profesor Calvo fue esencial para el nacimiento y la posterior consolidación del LSJUZ. Aportó, no solo su entusiasmo y su gran capacidad de trabajo, sino su experiencia y los estrechos lazos fortalecidos en el IISJ que favorecieron la internacionalización del LSJUZ, su visibilidad y facilitaron el contacto, siempre enriquecedor, con la comunidad científica internacional que se articula a través del IISJ en torno a la sociología jurídica.

Pero una institución es sobre todo las personas que la integran, que le dan vida y sentido. Y Manuel Calvo supo también construir un equipo con compañeros y compañeras de diferentes países, a ambos lados del Atlántico (Brasil, Perú, Colombia, Argentina o España), más o menos permanentes. Investigadores e investigadoras a las que siempre apoyó y cuidó, por los que se preocupaba y a los integró en proyectos más allá de la realización de una tesis doctoral. ${ }^{8}$ El Laboratorio se convirtió, en manos de Manuel, en

\footnotetext{
6 Puede visitarse la página web del Laboratorio de Sociología Jurídica en el siguiente enlace: https://sociologiajuridica.unizar.es [con acceso el 20 de abril de 2020].

${ }^{7}$ Para más información acerca de esta red, puede visitarse la siguiente página web. El tiempo de los derechos. https://redtiempodelosderechos.com [con acceso el 20 de abril de 2020].

${ }^{8}$ El Profesor Calvo dirigió y codirigió numerosas tesis doctorales, a las que dedicó grandes esfuerzos y a las que daba mucha importancia en su trabajo, sobre una amplia variedad de temas y fenómenos como, apenas a modo de ejemplo y sin ánimo exhaustivo: el tratamiento jurídico de la pobreza, infancia y adolescencia en conflicto con la norma, presupuesto participativo, derechos de los niños/as y adolescentes en Brasil, populismo penal, gobierno de la infancia, secularización y pluralismo religioso, percepción del riesgo, garantías y tratamiento jurídico del accidente de trabajo en el sector de la construcción, el derecho a la
} 
un espacio de discusión, de debate, en un ámbito donde desarrollar investigaciones, colaborar en proyectos; en definitiva, hacer sociología jurídica tal y como él entendía debía hacerse. ${ }^{9}$

Dentro de la línea principal de investigación del LSJUZ que concierne a la "[i]mplementación y efectividad de los derechos humanos con una especial referencia a los sociales", los trabajos empíricos, informes y publicaciones han sido incesantes desde hace más de veinte años. La vinculación del LSJUZ con el Proyecto El tiempo de los derechos (CSD2008-0007, 2008-2014) supuso un importantísimo impulso a nivel nacional e internacional. El principal objetivo de esta línea de investigación ha sido y sigue siendo llevar a cabo un análisis integral del papel de los derechos humanos en las sociedades contemporáneas, identificando retos, y desafíos teóricos y prácticos, pero también, proponiendo vías de solución de conduzcan al fortalecimiento del Estadosocial y de Derecho.

Además de la elaboración de numerosos estudios e informes, ${ }^{10}$ el LSJUZ viene realizando desde su creación jornadas abiertas y congresos anuales dedicados al estudio académico de diversos aspectos jurídicos y sociológicos relativos a los derechos humanos. Dentro de estos, como veremos después con más detalle, se ha dado una especial importancia al problema de la violencia de género. Asimismo, el LSJUZ organizó en 2018 un congreso internacional sobre teoría de la legislación, que reunió a importantes expertos europeos y españoles en materia de legisprudencia y calidad legislativa. Este mismo año, con fecha de 5 de febrero se dio comienzo a un Seminario abierto a la Facultad de Derecho, a la Universidad de Zaragoza y al público en general en el que, con periodicidad mensual, se va a dar cuenta de las principales novedades jurídicas y científicas relativas a los temas de investigación más relevantes para el Laboratorio: violencia de género, violencia a los mayores, victimología, igualdad de oportunidades y dificultades en el acceso a la justicia de los derechos sociales, económicos y culturales, como también puede observarse en el enlace antes indicado. ${ }^{11}$

\footnotetext{
información del consumidor, estrategias de promoción de la igualdad en el ámbito universitario, la implementación del derecho humano a la alimentación adecuada en Brasil, formulación de políticas públicas sociales, educación ambiental, o la construcción social del principio constitucional de la protección en Brasil. Todos estos trabajos dan muestra de la amplitud de intereses del Profesor Calvo y también del notable éxito que tuvo a la hora de convencer, como veíamos instaba en los 90 (Calvo 1995, 39), a jóvenes investigadores/as que empezaban su carrera a escribir sus tesis doctorales en el ámbito de la sociología jurídica.

${ }^{9}$ Como prueba de la capacidad de trabajo y de la amplitud de intereses con las que Manuel Calvo estructuró el LSJUZ, podemos presentar las tres líneas de investigación seguidas en los últimos proyectos del LSJUZ: Implementación y efectividad de los derechos humanos (con especial referencia a los derechos sociales); Análisis crítico del proceso de justificación de leyes: calidad e impacto efectivo de la legislación en un contexto globalizado; y, finalmente, Implementación y eficacia de la LO 1/2004, de 28 de diciembre de Protección Integral contra la Violencia de Género: Evaluación y propuestas de intervención preventiva, de la que hablaré en el siguiente apartado al referirme a las investigaciones sobre violencia degénero.

${ }^{10}$ Debemos referirnos también a la colección de artículos LSJ Working Papers y a otras publicaciones que junto a algunos informes de investigación y estudios se encuentran disponibles en la ya citada página web del LSJUZ.

11 Estas breves notas acerca del LSJUZ han sido enriquecida y completadas por la información proporcionada por la Profesora María José González Ordovás, actual directora del LSJUZ, ha tomado el relevo y la responsabilidad de encabezar el equipo de miembros y colaboradores/as y continuar con la labor iniciada por el Profesor Calvo.
} 


\section{La violencia de género como prioridad en la investigación socio-jurídica}

Entre los variados temas que ocuparon y preocuparon a Manuel Calvo, la violencia ejercida contra las mujeres ocupó siempre un lugar relevante. La violencia de género se ha ido convirtiendo, en los últimos años e incluso décadas, en una preocupación que ha llegado hasta las agendas políticas y académicas, entendiéndose como un asunto al que se deben dedicar esfuerzos estructurados, sostenidos y coordinados dirigidos tendencialmente hacia su erradicación.

En esta línea, desde el inicio del LSJUZ, fueron varias los trabajos de investigación que abordaron esas cuestiones relacionadas con la violencia de género, analizando la naturaleza y alcance del fenómeno, investigando la implementación de las normas, y en algún sentido, también aportando propuestas y una base empírica para la toma de decisiones en un periodo de tiempo en el que se sucedieron reformas legislativas y se emprendieron políticas públicas en España con la intención de combatir decididamente los efectos de una realidad que, hasta entonces, había permanecido confinada en el ámbito de lo privado y con una visibilidad social limitada. El abordaje que desde el LSJUZ se ha venido realizando de la violencia de género ha abarcado, en estos años, tanto la perspectiva teórica como sus implicaciones prácticas, sin descuidar lo relativo a la sensibilización entre los universitarios y los no universitarios ante dicho problema.

Desde el punto de vista teórico, entre su amplia producción científica sobre el tema, tenemos que destacar varios análisis críticos realizados por Manuel Calvo a partir de la entrada en vigor de la LO 1/2004 de 28 de diciembre, de Medidas de Protección Integral contra la Violencia de Género. Por ejemplo, en un artículo publicado en 2016, tras un análisis pormenorizado de los antecedentes legislativos y del contenido de la LO 1/2004, concluía que la alternativa de una ley Integral alternativa parece "la más adecuada para afrontar un problema de raíces complejas y soluciones que desbordan la mera intervención penal. La respuesta jurídica frente a la violencia familiar de género debe contemplar objetivos de transformación social profunda y de prevención dirigidos a erradicar de la violencia de género; de intervención eficaz frente a las situaciones de riesgo; y de protección efectiva -e integral- de las víctimas como último recurso" (Calvo 2006 , p. 129). Aunque igualmente advertía que "la etiqueta que le pongamos a una ley no resuelve por sí misma todos los problemas de un fenómeno social tan enraizado y de dimensiones tan amplias como el de la violencia de género. Una Ley Integral debe serlo tanto en su contenido como -sobre todo- en la práctica, esto es, mediante la implementación de políticas públicas tendentes a la prevención y al control de la violencia familiar de género, dotadas con los recursos humanos y materiales pertinentes para alcanzar los objetivos previstos" (Calvo 2006, 129-130). Esto es, para evitar convertir la ley en papel mojado, "será necesario crear una estructura de implementación operativa suficientemente de recursos humanos y materiales; desarrollar mecanismos de control positivo y negativo apropiados; y promover la evaluación continua de los procesos desarrollados y los objetivos alcanzados" (Calvo 2006, 129-130). No obstante, en un reciente análisis, con más de una década de perspectiva, defendía que, a pesar de la intervención penal no ser la solución al problema, tampoco se debía prescindir de la misma debido a su fuerte poder simbólico:

We do not believe that police intervention and criminal proceedings are enough to eradicate gender violence. It is clear that this is not reasonable, but we should also ask 
ourselves whether we should dispense with it. Perhaps the response here should also be negative. It is true that reformism in criminal proceedings and progress in punishment will not definitively resolve the objectives aimed at eradicating gender violence. However, criminal law has a symbolic power that we should not underestimate. (Calvo 2019, p. 256)

Es obvio que consideraba que la investigación socio-jurídica podía prestar un apoyo considerable en esa tarea de evaluación continua tal y como demuestran los diferentes proyectos de investigación abordados desde el LSJUZ y también varias de las tesis doctorales que dirigió y codirigió en relación con este tema. ${ }^{12}$

Otra línea teórica desarrollada por el Profesor Calvo en algunos de sus últimos trabajos sobre violencia de género ahondaba en el abordaje desde una perspectiva de derechos humanos y, específicamente, en el papel de los movimientos sociales y del feminismo en el reconocimiento a un derecho de las mujeres a vivir libres de violencia de género. En este sentido, su posición era clara:

Gender violence is certainly a crime, but it is much more than an offence. Therefore, if this social scourge is to be eradicated, it is essential to speak of it in the language of rights. Dealing with the problem in terms of human rights is especially productive because it provides a broader perspective without ignoring the advances implicit in criminal and other legal reforms. (Calvo 2016, p. 74)

Ese lenguaje de los derechos humanos, en opinión del Profesor Calvo podía contribuir a fortalecer las acciones de los movimientos de mujeres contra la violencia de género. En sus propias palabras:

A more complex analysis of the mechanisms of social power and the design, on the part of the women's movement and organizations, of more wide-reaching actions of social intervention complementary to state intervention may perhaps result in a more productive strategy in terms of social change. (Calvo 2016, p. 74)

Desde el punto de vista empírico, son varios los proyectos de investigación en esta área que, encabezados por Manuel Calvo como investigador principal, fueron financiados por diferentes administraciones e instituciones.

Por nombrar apenas algunos trabajos concretos, destacan las pioneras y amplias investigaciones realizadas para el CGPJ, a través del análisis de sentencias y otros documentos del proceso penal correspondientes a los años 1999 (Calvo 2003) ${ }^{13}$ y 2000-

\footnotetext{
${ }^{12}$ Entre estas tesis podemos destacar las de Laura Gómez (sobre menores víctimas y testigos de violencia de género); Mariana Sánchez (género y derecho); Jorge Gracia (sobre maltrato familiar hacia las personas mayores con una especial atención a la violencia de género hacia mujeres mayores) o, más recientemente, Paz Olaciregui (prevenir la violencia de género: análisis de las herramientas de valoración y gestión del riesgo), Elena Pilcher (la violencia contra las mujeres a través del derecho, las instituciones y el movimiento feminista)y, la todavía en proceso de realización, de Alicia Brox (sobre el Convenio de Estambul y la perspectiva de género en relación con las violencias contra las mujeres en el derecho francés y español).

${ }^{13}$ Este estudio, realizado para el Consejo General del Poder Judicial, parte de una amplia investigación (4.648 registros realizados en Juzgados de lo Penal y Juzgados de Instrucción y la totalidad de las sentencias de las Audiencias provinciales, Tribunales Superiores de Justicia y Tribunal Supremo) que permitió obtener datos significativos sobre el conjunto del Estado respecto del tratamiento en ese momento de la violencia intrafamiliar ante la Administración de Justicia y las características socio-demográficas de las víctimas y los agresores. El estudio mencionado tenía dos partes diferenciadas que siguen vertebrando estas reflexiones. La primera consistió en la recogida y análisis de datos procedentes de Juzgados de Instrucción y Juzgados de lo Penal. Y la segunda, se centró en las sentencias de las Audiencias provinciales dadas en primera
} 
2002 (Calvo 2004); ${ }^{14}$ también a los estudios e investigaciones realizados para el Instituto Aragonés de la Mujer (IAM). Es el caso de la investigación que concluyó con la elaboración del Informe sobre la Violencia de Género en Aragón (Calvo et al. 2012) ${ }^{15}$ o el estudio Menores víctimas de Violencia de Género en Aragón 2010-2012 (Calvo et al. 2013). ${ }^{16}$

Estas investigaciones muestran la enorme capacidad de trabajo del Profesor Manuel Calvo, al que, como se suele decir, no se le ponía nada por delante. Ya fuera la necesidad como jurista de adquirir herramientas de sociólogo, o el afrontar la burocracia, la incomodidad y las preocupaciones que implicaban la opción por una sociología jurídica empírica, que traía aparejada muchas veces diseños de investigación con complejos trabajos de campo. El Profesor Calvo siempre fue coherente con su idea de sociología jurídica y ahí está su aportación científica para demostrarlo. Nunca se quedó en la comodidad de su despacho, sino que, como a veces le gustaba decir, había que ensuciarse las manos y, por ello, descendió con rigor y también con un entusiasmo que contagiaba a todos los que estábamos cerca, al barro de la investigación empírica.

En la actualidad, la violencia de género sigue siendo una de las líneas de investigación prioritarias del LSJUZ. Recientemente la actividad investigadora del LSJUZ en esta materia se ha encauzado a través del proyecto de investigación El tratamiento de la violencia de género en la Administración de Justicia. Implementación y eficacia de la LO 1/2004 (DER2014-55400-R) que tuvo como objetivo general evaluar la eficacia y efectividad de las reformas en materia de violencia de género que culminaron con la entrada en vigor de la citada ley.

Dentro de esa atención permanente a la violencia de género, coherentemente con la concepción de una sociología jurídica reflexiva que defendía Manuel Calvo, ocupan un papel esencial las Jornadas nacionales que anualmente ha venido celebrando el LSJUZ sobre violencia doméstica desde 2016. En 2020 se debían haber celebrado las VI Jornadas, pero en este año aciago, tuvieron que ser sustituidas el 26 de noviembre por la Jornada celebrada online Diálogo: 20 años no es nada. Pasado, presente y futuro de la respuesta ante la violencia de género. Este Diálogo entre la Profesoras Encarna Bodelón, de la Universidad

instancia -incluidas las de los Tribunales de Jurado- y las resolutorias de recursos; así como las sentencias de los Tribunales Superiores de Justicia y el Tribunal Supremo.

${ }^{14}$ El estudio del LSJUZ (Calvo 2004) seguía una metodología similar partiendo de una amplia recogida de datos de sentencias y autos y otras fuentes documentales (correspondientes a los años 2000, 2001 y 2002) ligadas al procedimiento y a su ejecución. La búsqueda de datos se realizó en casos relativos a la variada tipología delictiva relacionada con la violencia intrafamiliar establecida de acuerdo con el concepto instrumental de violencia doméstica aprobado por el Observatorio de Violencia Doméstica en su reunión de 20 de noviembre de 2002 y los correspondientes criterios de identificación de resoluciones en materia de medidas cautelares, así como de sentencias dictadas en procedimientos sobre violencia doméstica. De esta forma se obtuvieron un total de 11.924 registros en toda España.

15 Se elaboró el mismo con los datos recogidos correspondientes al año 2011 partiendo de los Indicadores para conocer y profundizar sobre la violencia de género en Aragón, diseñados inicialmente por el elaborado por el Grupo de Trabajo formado con ese objetivo dentro del Observatorio Aragonés de Violencia sobre la Mujer (Calvo et al. 2012).

${ }^{16}$ Los objetivos de este trabajo buscaban fundamentalmente identificar y hacer aflorar el alcance y las características del fenómeno de la violencia de género en los menores que son víctimas directas e indirectas de violencia de género. Además, la investigación perseguía conocer y reflexionar sobre la implantación de los mecanismos de protección judicial y social de los hijos e hijas de mujeres víctimas de género, profundizando en particular sobre cómo se desarrolla el derecho a la Asistencia Social Integral de los menores sujetos de medidas de protección judicial en la Comunidad Autónoma de Aragón. 
Autónoma de Barcelona, y Juana María Gil Ruiz, de la Universidad de Granada, se convirtió en una oportunidad para revisar críticamente los cambios y también las perspectivas de futuro en relación con las políticas públicas entorno a la violencia de género y en una manera de rendir homenaje a la figura del Profesor Calvo García a través de la participación de colegas de otras universidades que siempre colaboraron estrechamente con el Laboratorio.

Creo que no me equivoco afirmando que Manuel Calvo estaba especialmente orgulloso de estas Jornadas que anualmente ha venido celebrado el LSJUZ sobre violencia de género y que constituyen una muestra de sus resultados científicos además de su transferencia a la sociedad. A lo largo de estos años hemos contado con participantes procedentes de ámbitos institucionales, académicos y profesionales relacionados con la violencia de género, buscando un enfoque plural e interdisciplinar que aportaron su conocimiento como participantes en conferencias, mesas y debates o presentando numerosas comunicaciones. Estas jornadas ejemplifican muy bien su idea de la sociología jurídica: no encerrada en los muros de la academia sino abierta a la sociedad; empírica sin dejar de lado la teoría; rigurosa, pero comprometida con la transformación social y la realización de los derechos humanos.

\section{5. ¿Cómo lo haría Manolo?}

En estos apuntes he tratado de ordenar algunas ideas sobre la aportación de Manuel Calvo en el desarrollo de la sociología jurídica en España. Creo que esta breve descripción muestra una trayectoria coherente y comprometida. Desde luego, elProfesor Calvo hizo mucho más en este campo que dedicarse a trabajar por las tardes en la sociología jurídica, como hemos visto decía a mediados de los 90, con una sorna que le era tan propia. Manuel reflexionó con profundidad y hondura teórica sobre la disciplina, con el rigor conceptual e intelectual que le caracterizaba, y además apostó por realizar investigación empírica en consonancia con su propia concepción de lo que la sociología jurídica debería ser. Su contribución fue realizada desde diferentes ámbitos -la docencia y la investigación- y espacios -como el Instituto Internacional de Sociología Jurídica de Oñati o el Laboratorio de Sociología Jurídica de la Universidad de Zaragoza- que supo conectar e integrar. E investigó cuestiones relevantes para, desde el mayor rigor y exigencia, favorecer la transformación social y la profundización de los derechos humanos, como demuestra por ejemplo su interés en la violencia de género. También consiguió, no solo convencer a jóvenes investigadores para que hiciéramos nuestras tesis en el campo de la sociología jurídica, sino dejar profunda huella, personal y académica, a ambos lados del Océano Atlántico entre colegas de España, Argentina, Colombia o Brasil que tuvimos la suerte y el privilegio de trabajar a su lado.

En el imponente museo del cine de Berlín, que se centra en la relevante aportación alemana a la historia del cine, se exhibe en una vitrina, junto con otras de sus pertenencias, un pequeño cartel que se encontraba colgado originariamente en el despacho de Billy Wilder. En él se puede leer la siguiente inscripción: How would Lubitsch do it? (¿Cómo lo haría Lubitsch?). Wilder aseguraba que siempre que se encontraba con alguna dificultad en el desarrollo de sus proyectos, se le atascaba un guion o un rodaje no marchaba como debía, si estaba cerca, iba un rato al despacho y miraba el cartel que le preguntaba cómo lo haría su maestro. Necesitaba pensarlo, aunque no necesariamente para estar de acuerdo o acatar una autoridad incuestionada. Simplemente sentía ese impulso, 
lamentablemente imposible de satisfacer en persona tras su desaparición, de intercambiar puntos de vista, de saber lo que él pensaría, de valorar inquietudes y templar vacilaciones. Aunque sólo pueda hablar desde mi propia experiencia, me parece que siempre tendré en mi interior, aunque sea de forma simbólica, un cartel semejante al que tenía Wilder en su despacho, que me hará constantemente preguntarme: ¿Cómo lo haría Manolo?

\section{Referencias}

Arrese Murguzur, S., y Pérez-Perdomo, R., 2016. El Máster de Sociología Jurídica de Oñati: la percepción de los graduados. Oñati Socio-Legal Series [en línea], 6(3), 901919. Disponible en: http://ssrn.com/abstract=2676532 [Con acceso el 21 de abril de 2021].

Calvo García, M., 1995. La investigación socio-jurídica en España: estado actual y perspectivas. En: R. Bergalli et al., eds., El Desarrollo y las aplicaciones de la Sociología Jurídica en España. Oñati: IISJ, col. Oñati Proceedings noำ19, 17-46.

Calvo García, M., 2003. El tratamiento de la Violencia de Género en la Administración de Justicia [en línea]. Madrid: Centro de Documentación del Consejo General del Poder Judicial. Disponible en:

https://www.poderjudicial.es/cgpj/ca/Temes/Funcionament-delsTribunals/Percepcion-social-de-la-justicia/El-tratamiento-de-la-violenciadomestica-en- la-administracion-de-justicia [Con acceso el 21 de abril de 2021].

Calvo García, M., 2004. La violencia doméstica y su tratamiento en la Administración de Justicia. LSJUZ y CGPJ. Madrid: CGPJ.

Calvo García, M., 2006. Análisis socio-jurídico de la Ley Orgánica de medidas de protección integral contra la violencia de género. Trabajo; Revista iberoamericana de relaciones laborales [en línea], nº 17, 105-131 Disponible en:

http://rabida.uhu.es/dspace/bitstream/handle/10272/2478/b15148932.pdf?sequenc $\mathrm{e}=1$ [Con acceso el 21 de abril de2021].

Calvo García, M., 2010. ¿Cabe el enfoque socio-jurídico en la teoría del derecho? Anales de la Cátedra Francisco Suárez [en línea], 44, 371-394. Disponible en:

http://revistaseug.ugr.es/index.php/acfs/article/view/512/602 [Con acceso el 21 de abril de 2021].

Calvo García, M., 2014. Elogio del doctorando Profesor Dr. Vincenzo Ferrari. Acto de Investidura del Grado de Doctor honoris Causa Vincenzo Ferrari. Prensas de la Universidad de Zaragoza, 7-18.

Calvo García, M., 2016. The Role of Social Movements in the Recognition of Gender Violence as a Violation of Human Rights: From Legal Reform to the Language of Rights. The Age of Human Rights Journal [en línea], no 6, 60-82. Disponible en: https://doi.org/10.17561/tahrj.v0i6.2930 [Con acceso el 21 de abril de 2021].

Calvo García, M., 2019. The Treatment of Gender Violence in the Spanish Administration of Justice: Implementation and Effectiveness of the Organic Act 1/2004, on Integral Protection Measures Against Gender Violence. US-China Law 
Review [en línea], 16(6), 237-257. Disponible en: http://dx.doi.org/10.17265/15486605/2019.06.003 [Con acceso el 21 de abril de 2021].

Calvo García, M., et al., 2012. La violencia de género en Aragón [en línea]. Dirigido por M. Calvo García y C. Mesa Raya. LSJUZ e IAM. Disponible en:

https://www.aragon.es/documents/20127/674325/indicadores1.pdf/ef96682b-388e9a3e-4856-ab1f2ef09259 [Con acceso el 21 de abril de 2021].

Calvo García, M., et al., 2013. Menores víctimas de violencia de género en Aragón: 2010-2012 [en línea]. Dirigido por M. Calvo García y C. Mesa Raya. LSJUZ e IAM.

Disponible en:

https://www.aragon.es/documents/20127/674325/Nov\%202013 Menores\%20victi mas.. pdf/d8e10d73-8def-2d0c-a5dc-22289fa7a5e3 [Con acceso el 21 de abril de 2021].

Calvo García, M., y Picontó Novales, T., 2017. Introducción y perspectivas actuales de la sociología jurídica. Barcelona: UOC.

Instituto Internacional de Sociología Jurídica (IISJ), 2020. In memoriam Manuel Calvo García (1956-2020) [en línea]. Disponible en: http://www.iisj.net/es/sobre-eliisj/actualidad/memoriam-manuel-calvo- garc\%C3\%ADa-1956-2020 [Con acceso el 21 de abril de 2021].

Melville, A., y Arrese Murguzur, S., 2016. Perceptions of Teachers at the International Institute of Sociology of Law of International Student Diversity: Barriers, Enrichmentor Cosmopolitan Learning? Oñati Socio-Legal Series [en línea], 6(3), 607-631. Disponible en: http://ssrn.com/abstract=2805941 [Con acceso el 21 de abril de 2021].

Toharia. J.J., 1975. El juez español: Un análisis sociológico. Madrid: Tecnos.

Toharia. J.J., 1988. Los españoles ante la Administración de Justicia. Madrid: CIS.

Toharia. J.J., 1994. Actitudes de los españoles ante la Administración de Justicia. Madrid: CIS.

Treves, R., 1988. La sociología del derecho: Orígenes, investigaciones, problemas. Barcelona: Ariel. 